\title{
Endowing Polymeric Assemblies with Unique Properties and Behaviors by Incorporating Versatile Nanogels in the Shell
}

Leilei Lv, Zhen Zhang, Huiya Li, Xiayun Huang*, Daoyong Chen*

State Key Laboratory of Molecular Engineering of Polymers, Department of Macromolecular Science, Fudan

University, 200438, Shanghai, China

\section{Table of Contents}

Table of Contents 1

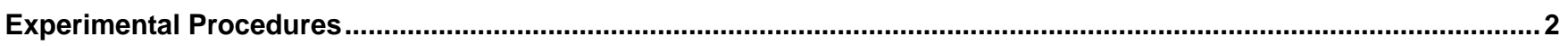

S1 Definition of Geometric Packing Parameter $(P)$....................................................................................................

S2 Accumulated Repulsion from a Large Number of $c$-P4VP Nanogels Densely Distributed in the Shell of PW or Vesicle can be Strong Enough to Break the Assembly with a Frozen Core .................................................................... 3

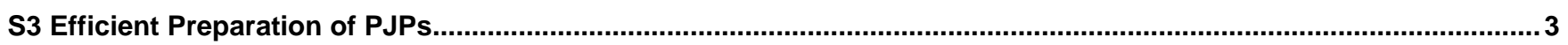

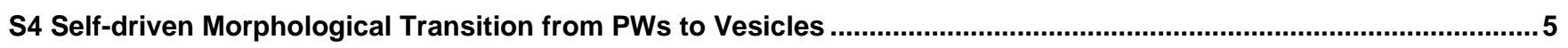

S5 Worm-to-Vesicle Transition Observed by Cryogenic Electron Microscopy .............................................................5

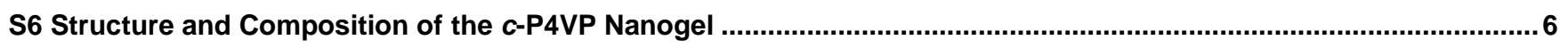

S7 Self-driven Morphological Transition from Spherical Micelles to Vesicles ............................................................... 7

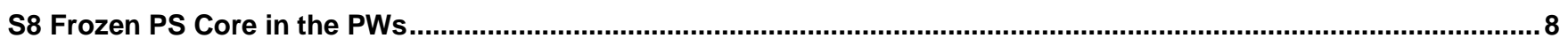

S9 Existence of Excess TFAs has No Influence on the Results of the Present Study ............................................... 8

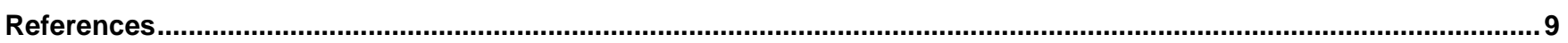




\section{Experimental Procedures}

\section{Materials and Characterization}

Poly(styrene-block-methyl methacrylate) $\left(\mathrm{PS}_{528}-b-\mathrm{PMMA}_{220}\right.$; abbreviated as $\left.\mathrm{SM}, M_{\mathrm{w}} / M_{\mathrm{n}}=1.09\right)$ and poly(styrene-block-4vinylpyridine) $\left(\mathrm{PS}_{480}-b-\mathrm{P}_{4 \mathrm{VP}} \mathrm{P}_{124}\right.$; abbreviated as $\left.\mathrm{SV}, M_{\mathrm{w}} / M_{\mathrm{n}}=1.15\right)$ diblock copolymers were purchased from Polymer Source Inc. 1,4dibromobutane (DBB) was purchased from Sigma-Aldrich and used directly. N, N-dimethylformamide (DMF) and tetrahydrofuran (THF) were purchased from Sinopharm Chemical Reagent Co., Ltd and purified through a PS-MD-3 solvent purification system (Innovative Technology Inc., America) for moisture removal. Methanol $(\mathrm{MeOH})$ and silver nitrate $\left(\mathrm{AgNO}_{3}\right)$ were purchased from Sinopharm Chemical Reagent Co., Ltd. and used without further purification. Trifluoroacetic acid (TFA) from Aladdin was used as received.

Dynamic light scattering (DLS) was carried out using an ALV-5000 laser light scattering spectrometer. All sample solutions were filtered through $450 \mathrm{~nm}$ filters and measured at $25 \pm 0.1^{\circ} \mathrm{C}$. The hydrophobic Millex-FH filter was selected when using THF as the solvent, while the hydrophilic Millex-LCR filter was selected when using MeOH/THF mixed solvent as the solvent. He-Ne laser was utilized as the light source with a wavelength of $632 \mathrm{~nm}$. DLS measurement was carried out at a scattering angle of $90^{\circ}$. The hydrodynamic radius, $\left\langle R_{h}\right\rangle$, was calculated using the Stokes-Einstein relation via the CONTIN program. Transmission electron microscopy (TEM) observations were carried out by the FEI Tecnai G2 20 TWIN at the acceleration voltage of $200 \mathrm{kV}$ for the high contrast sample or the Philips CM120 at the acceleration voltage of $80 \mathrm{kV}$ for the low contrast sample. During sample preparation, $1.5 \mu \mathrm{L}$ of sample solution was drop-casted onto the glow-discharged carbon-coated copper grids. The excess solution was blotted and removed using the filter paper. The TEM grid was then dried in the vacuum desiccator immediately. Samples for cryogenic electron microscopy were prepared using FEI Vitrobot MKIV to avoid solvent loss from the solution during sample preparation. The vitrified samples were stored under liquid nitrogen, and subsequently transferred to the Gatan 626 cryoholder and observed by electron microscope (Tecnai G2 20). Images were collected at a temperature of $-170{ }^{\circ} \mathrm{C}$ and $200 \mathrm{kV}$ acceleration voltage. Gel permeation chromatography (GPC) was conducted on Agilent 1260 GPC analysis system comprising two Polymer Laboratories PL gel $5 \mu \mathrm{m}$ Mixed-C columns connected in series to a 390-MDS multidetector suite. The refractive index detector was used. DMF with $1.6 \mathrm{~g} / \mathrm{L} \mathrm{LiBr}$ was chosen as the eluent. The flow rate and operating temperature were fixed at $1.0 \mathrm{~mL} / \mathrm{min}$ and $50{ }^{\circ} \mathrm{C}$, respectively. ${ }^{1} \mathrm{H}$ nuclear magnetic resonance ( ${ }^{1} \mathrm{H}$ NMR) spectra were measured at $25^{\circ} \mathrm{C}$ on a $400 \mathrm{MHz}$ Brucker AVANCE III HD NMR spectrometer and analyzed with Bruker Topspin software using tetramethylsilane (TMS) as a reference.

\section{Preparation of Polymeric Janus Particles (PJPs)}

Firstly, SVISM mixed shell micelles (MSMs) were prepared. In brief, $2.5 \mathrm{mg}$ SV and $2.5 \mathrm{mg}$ SM diblock copolymers were added to $10 \mathrm{~mL}$ of THF and stirred for $48 \mathrm{~h}$ to ensure the complete dissolution. The SV/SM MSMs were made by dropwise adding $15 \mathrm{~mL}$ of $\mathrm{MeOH}$ to $10 \mathrm{~mL}$ of the SV/SM diblock copolymer solutions with stirring. The volume ratio of MeOH/THF in the SV/SM MSM suspension was 1.5/1. Subsequently, the P4VP blocks in the mixed shell of MSMs were intramicellarly cross-linked by using DBB. DBB was added to the SVISM MSMs suspension. The molar ratio of DBB/4VP is 10:1. The mixture was then continuously stirred at $20^{\circ} \mathrm{C}$ for $60 \mathrm{~h}$ and at room temperature for $40 \mathrm{~h}$ to obtain the cross-linked mixed shell micelles (c-MSMs). After that, $25 \mathrm{~mL}$ of $\mathrm{c}$ MSMs suspension was dialyzed against $1000 \mathrm{~mL}$ THF or DMF and refreshed 3 times for every $12 \mathrm{~h}$ to ensure complete dissociation of $c$-MSMs. During dialysis, the unreacted DBB in the suspension was removed entirely, and the $c$-MSMs were dissociated into polymeric Janus particles (PJPs) and molecularly dissolved SM. The suspension was then concentrated to $10 \mathrm{~mL}$ for the subsequent coassembly.

\section{Staining PJPs with $\mathrm{AgNO}_{3}$ for TEM Observation}

The staining of PJPs was conducted by adding $1.0 \mathrm{mg}$ of $\mathrm{AgNO}_{3}$ to the $2.0 \mathrm{~mL}$ of dissociated $c-M S M s$ suspension in DMF. The mixture was stirred in the dark at room temperature for $24 \mathrm{~h}$. Excess insoluble $\mathrm{AgNO}_{3}$ was removed by centrifugation at $3000 \mathrm{rpm}$ for $15 \mathrm{~min}$, and the supernatant was collected for the TEM observation. DMF was selected as the solvent since the solubility of $\mathrm{AgNO}_{3}$ in DMF is better than that in THF so that PJPs have a better staining performance in DMF. The switch of solvent between THF and DMF does not affect the composition and structure of PJPs.

\section{Preparation of Polymeric Worm-like Micelles (PWs) and their Morphological Transition to Vesicles}

The polymeric worm-like micelles (PWs) were prepared by co-assembling the equal amount of PJPs $(0.25 \mathrm{mg} / \mathrm{mL})$ and molecularly dissolved SM $(0.25 \mathrm{mg} / \mathrm{mL})$ in $\mathrm{MeOH} / \mathrm{THF}(1.5 / 1, \mathrm{v} / \mathrm{v})$ mixed solvent. It was conducted by dropwise adding $1.5 \mathrm{~mL}$ of $\mathrm{MeOH}$ to $1 \mathrm{~mL}$ of dissociated c-MSMs suspension in THF with stirring. After the suspension of PWs in MeOH/THF (1.5/1, v/v) mixed solvent was sealed in the sintered ampoule glass bottle, the PWs in the suspension were slowly and steadily converted to vesicles and all the PWs were converted into vesicles after 28 days, which was tracked by TEM observation.

\section{Preparation of Spherical Micelles and their Morphological Transition to Vesicles}

$2.2 \mathrm{mg} \mathrm{SV}$ and $2.0 \mathrm{mg} \mathrm{SM}$ diblock polymer were added into $8.4 \mathrm{~mL}$ of THF and stirred for $48 \mathrm{~h}$ to ensure the complete dissolution. Subsequently, $12.6 \mathrm{~mL}$ of $\mathrm{MeOH}$ was dropwise added to $8.4 \mathrm{~mL}$ of the SV/SM diblock copolymer solutions with stirring The SV/SM MSMs were obtained in MeOH/THF $(1.5 / 1, \mathrm{v} / \mathrm{v})$ mixed solvent and used for the subsequent cross-linking of P4VP blocks on the mixed shell. Then, DBB was added to the SVISM MSMs suspension. The molar ratio of DBB/4VP is 10:1. The mixture was continuously stirred at $-20^{\circ} \mathrm{C}$ for $60 \mathrm{~h}$ and at room temperature for $40 \mathrm{~h}$ to obtain the $c$-MSMs. By the dialysis against THF, $c$-MSMs were dissociated into PJPs and molecularly dissolved SM. After dialysis, the suspension was concentrated to $8.4 \mathrm{~mL}$ for the subsequent coassembly. During coassembly, $12.6 \mathrm{~mL}$ of $\mathrm{MeOH}$ was dropwise added to $8.4 \mathrm{~mL}$ of the suspension with stirring. The spherical micelles with a diameter of $44 \pm 1 \mathrm{~nm}$ were obtained. After sealing the suspension in the sintered ampoule glass bottle, the 
spherical micelles in the suspension were gradually converted to the mixture of short PWs and vesicles, and all converted into vesicles after 40 days.

\section{Preparation of Core-frozen PWs/Vesicles and the Dissociation by TFA}

The core-frozen PWs and vesicles were firstly prepared by quickly adding $900 \mu \mathrm{L}$ of $\mathrm{MeOH}$ to $100 \mu \mathrm{L}$ of the corresponding suspension. The volume ratio of $\mathrm{MeOH}$ and THF was $24 / 1$. Then, $60 \mu \mathrm{L}$ of TFA was added into $1 \mathrm{~mL}$ of the suspension. The mixture was then stirred for $24 \mathrm{~h}$.

\section{S1 Definition of Geometric Packing Parameter $(P)$}

The geometrical packing parameter $(P)$ is defined as $P=v / a_{0} l_{c}$, where $a_{0}$ is the area of solvophilic domain while $v$ and $I_{c}$ are the volume and length of solvophobic domain, respectively. ${ }^{[1]}$ If $P<1 / 3$, micelle is obtained; if $1 / 3<P<1 / 2$, worm-like micelle is obtained; and if $1 / 2<P<1$, vesicle is obtained.

\section{S2 Accumulated Repulsion from a Large Number of $c$-P4VP Nanogels Densely Distributed in the Shell of PW or Vesicle can be Strong Enough to Break the Assembly with a Frozen Core}

In traditional polymeric assemblies, the shell is formed by linear polymer chains. When the core of the assemblies is in a frozen state, the repulsion between the shell-forming polymer chains induced by the complexation with the shell-forming polymer chains cannot induce an apparent morphological or structural variation. In these cases, the morphological transitions are prohibited because not only the mobility of the core-forming chains is seriously restricted, but also the shell-forming chains can largely change their conformation to relax the repulsion effectively.

In the present work, both the PWs and the vesicles have a PS core and a c-P4VP/PMMA mixed shell. To fully complex the $c$ P4VP, highly excess TFA was added to the suspension of frozen PWs or vesicles. The molar ratio of TFA/pyridine was 1000:1. As explained below (S2 in the $\mathrm{SI}$ ), due to the good solubility of TFA in the medium and its low boiling point, and the high immiscibility between PS and TFA, the uncomplexed TFA molecules existing in the system should have no considerable effect on the structure and behavior of the assemblies. Under these conditions, most of the unreacted pyridine groups were complexed with TFA. By the nearly complete complexation of the pyridine groups in the c-P4VPs with TFA, the size of the complexed $c$-P4VP was remarkably increased, resulting in the repulsion among the shell-forming components; the repulsion could be further enhanced due to the immiscibility between the fluorinated c-P4VP and the surrounding PMMA chains. It is noticeable that, as demonstrated in Figure $3 \mathrm{~b}$, there are a large number of $c$-P4VP nanogels densely distributed in the shell of an assembly. The repulsions from a large number of expanded nanogels in a same shell are accumulated and the accumulated repulsions could be strong enough. Compared with the traditional shell-forming components that are linear polymer chains and adopt a flexible conformation in the shell, the complexed $c$ P4VPs are much more difficult to compress or deform. As the strong accumulated repulsions are very difficult to be relaxed, the PWs or vesicles dissociated to relax the accumulated repulsions although the PS core was frozen in the medium at the MeOH/THF volume ratio of $24 / 1$

\section{S3 Efficient Preparation of PJPs}

\section{S3.1 Role of Relatively Long PMMA Chains in the Shell}

It has been reported that the shell in core-shell-corona micelles could be cross-linked intramicellarly even at a high concentration of the micelles. Due to the protection from the outermost corona layer, intermicellar cross-linking of the shell inside the corona layer was inhibited. ${ }^{[2]}$ In our previous study, ${ }^{[3]}$ the MSMs have a poly(butadiene) (PB) core and the PS/P4VP mixed shell. In the mixed shell, PS chains are longer than P4VP chains; the number of repeat units of PS is about 6 times that of P4VP. The location of the P4VP block in the MSMs is similar to the shell in the core-shell-corona micelles. The relatively long PS chains effectively prevented intermicellar cross-linking of P4VP. In this work, the PMMA chains in the mixed shell are also longer than that of P4VP chains; the number of repeat units of PMMA is about 1.8 times of 4VP in P4VP. Similarly, the P4VP chains are effectively protected by the long PMMA in the shell from intermicellar cross-linking. Confirmed by DLS and TEM (Figure 2), one SV/SM MSM was converted to one $c$ MSM and the cross-linking of P4VP occurred intramicellarly. 


\section{S3.2 The SM and PJPs Components in Dissociated c-MSMs Confirmed by GPC Characterization}

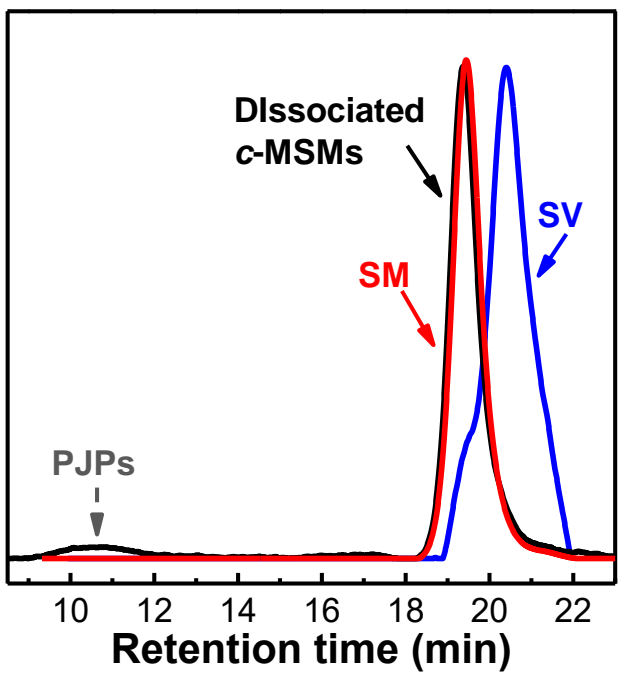

Figure S1 GPC curves of dissociated c-MSMs (black line), SV (red line) and SM (blue line) using DMF as the eluent.

After sufficient dialysis of $c$-MSMs suspension against THF, the common solvent of all components, the $c$-MSMs were expected to be dissociated to SM chains and PJPs. SM diblock polymer was completely soluble in THF. PJPs were composed by cross-linked P4VP (i.e. c-P4VP nanogel) and PS chains grafted on its one side.

The suspension of the dissociated $c-M S M s$ in THF was concentrated to ten times of the original concentration. Then the solvent was switched from THF to DMF for better characterizing by GPC, since DMF is a better solvent for the PJPs. In the GPC curve of dissociated $c$-MSMs, two peaks at the retention time of $10.6 \mathrm{~min}$ and $19.4 \mathrm{~min}$ were observed (Figure S1). The peak at the retention time of $10.6 \mathrm{~min}$ was assigned to the PJPs, and the peak at the retention time of $19.4 \mathrm{~min}$ was the signal of the molecularly dissolved SM. The GPC characterization also revealed that all the SV chains were converted to the PJPs because the signal of SV diblock polymer at 20.4 min disappeared in the GPC curve. We found that quaternized P4VP has a negative dn/dc value, which can explain why the signal of the PJPs in the GPC curve is very weak. The GPC characterization confirmed that $c$-MSMs successfully dissociated into individual SM chains and PJPs.

\section{S3.3 Staining the PJPs}

TEM observation is a powerful tool for characterizing small PJPs. The unstained $c$-P4VP nanogel in THF has very low contrast and cannot be clearly observed by TEM observation, and the PS chains are invisible in the images. Thus, the PJPs were stained for better observation by TEM in this work. Based on our previous study, staining from $\mathrm{AgNO}_{3}$ can enhance not only the contrast of the $c$-P4VP nanogel and PS domains but also the contrast difference between the two domains in the TEM images. ${ }^{[3]}$ Unfortunately, due to the extremely low solubility of $\mathrm{AgNO}_{3}$ in $\mathrm{THF}^{[4,5]}$ the staining of the PJPs in THF is unsuccessful. Therefore, we switched the solvent from THF to DMF, in which $\mathrm{AgNO}_{3}$ has better solubility than that in THF. ${ }^{[3]}$ Moreover, DMF is also the good solvent of both $\mathrm{SM}$ and the PJPs. TEM observation shows that the PJPs stained by $\mathrm{AgNO}_{3}$ in DMF has an asymmetric structure (Figure 2e). The high contrast domain is assigned to the $c$-P4VP nanogel (sensitive to the staining of $\mathrm{AgNO}_{3}$ ) and the low contrast domain the PS domains formed by multiple PS chains graft on one side of the $c$-P4VP. After stained by $\mathrm{AgNO}_{3}, \mathrm{SM}$ chains are still invisible in the TEM images because they exist as random coils in DMF and the chain density is too low. 


\section{S4 Self-driven Morphological Transition from PWs to Vesicles}

As shown in the TEM observation of the manuscript, when storing the sealed PW suspension, the PWs evolved slowly and steadily to the vesicles. Here, for each morphological evolution intermediate, more than 10 TEM images from different locations were taken and more than 100 assemblies were measured. Based on the statistical quantity and dimension of PWs and vesicles, the weight percentage of PWs and vesicles are calculated by the Equation 1 and 2, respectively. The density $(\rho)$ of PWs and vesicles are assumed to be the same. The radius of PWs and the wall thicknesses of vesicles are assumed to be the constant as $22.5 \mathrm{~nm}$ and $41 \mathrm{~nm}$, respectively.

$$
\begin{gathered}
m_{P W s}=\pi r^{2} \rho L \\
m_{\text {Vesicles }}=\pi D^{2} \rho d
\end{gathered}
$$

where $m_{P W s}$ and $m_{\text {Vesicles }}$ are the mass of PWs and vesicles, respectively. $r$ is the radius of PWs. $L$ is the average length of PWs. $D$ represents the average diameter of vesicles. And $d$ is the average wall thickness of the vesicles.

\begin{tabular}{|c|c|c|c|c|c|}
\hline & As-prepared & 3 days & 8 days & 15 days & 28 days \\
\hline Morphology & PW & PW+vesicle & $\mathrm{PW}+$ vesicle & PW+vesicle & Vesicle \\
\hline Weight Percentage of PWs & $100 \mathrm{wt} \%$ & $95 \mathrm{wt} \%$ & $\sim 51 \mathrm{wt} \%$ & $10 \mathrm{wt} \%$ & 0 wt $\%$ \\
\hline Weight Percentage of Vesicles & $0 \mathrm{wt} \%$ & $\sim 5 w t \%$ & $49 \mathrm{wt} \%$ & $90 \mathrm{wt} \%$ & $100 \mathrm{wt} \%$ \\
\hline Vesicle/PW Weight Ratio & 0 & 0.05 & 0.96 & 9.00 & - \\
\hline Average Length of PWs & $3.4 \mu \mathrm{m}$ & $3.0 \mu \mathrm{m}$ & $2.1 \mu \mathrm{m}$ & $1.2 \mu \mathrm{m}$ & - \\
\hline Average Diameter of Vesicles & - & $192 \mathrm{~nm}$ & $268 \mathrm{~nm}$ & $297 \mathrm{~nm}$ & $285 \mathrm{~nm}$ \\
\hline
\end{tabular}

Table S1 The parameters of PWs that undergo the morphological transition to vesicles obtained by TEM observation.

As listed in Table S1, initially, almost all the assemblies in the suspension had the PW morphology. The average length was 3.4 $\mu \mathrm{m}$. After the storing of 3 days, the weight percentage of PWs and vesicles were about $95 \mathrm{wt} \%$ and $5 \mathrm{wt} \%$, respectively. The average length of PWs decreased to $3.0 \mu \mathrm{m}$, and the formed vesicles had an average diameter of $192 \mathrm{~nm}$. After the sealed suspension was stored for 8 days, the suspension contained fewer PWs ( 51 wt $\%)$ and more vesicles ( 49 wt $\%)$. The vesicle/PW weight ratio increased to 0.96 . The average length of PW further decreased to $2.1 \mu \mathrm{m}$. The average diameter of vesicles increased to $268 \mathrm{~nm}$. When the storing time increased to 15 days, the PWs became even shorter; the average length of PW was $1.2 \mu \mathrm{m}$. The size of vesicles, however, did not have a distinguishable variation. Instead, the weight percentage of vesicles in the suspension increased to about $90 \mathrm{wt} \%$ and the vesicle/PW weight ratio increased to 9 . The PWs were completely converted to vesicles when the suspension was stored for 28 days. In the suspension, there were only vesicles. The average diameter of the vesicles was $285 \mathrm{~nm}$.

\section{S5 Worm-to-Vesicle Transition Observed by Cryogenic Electron Microscopy}
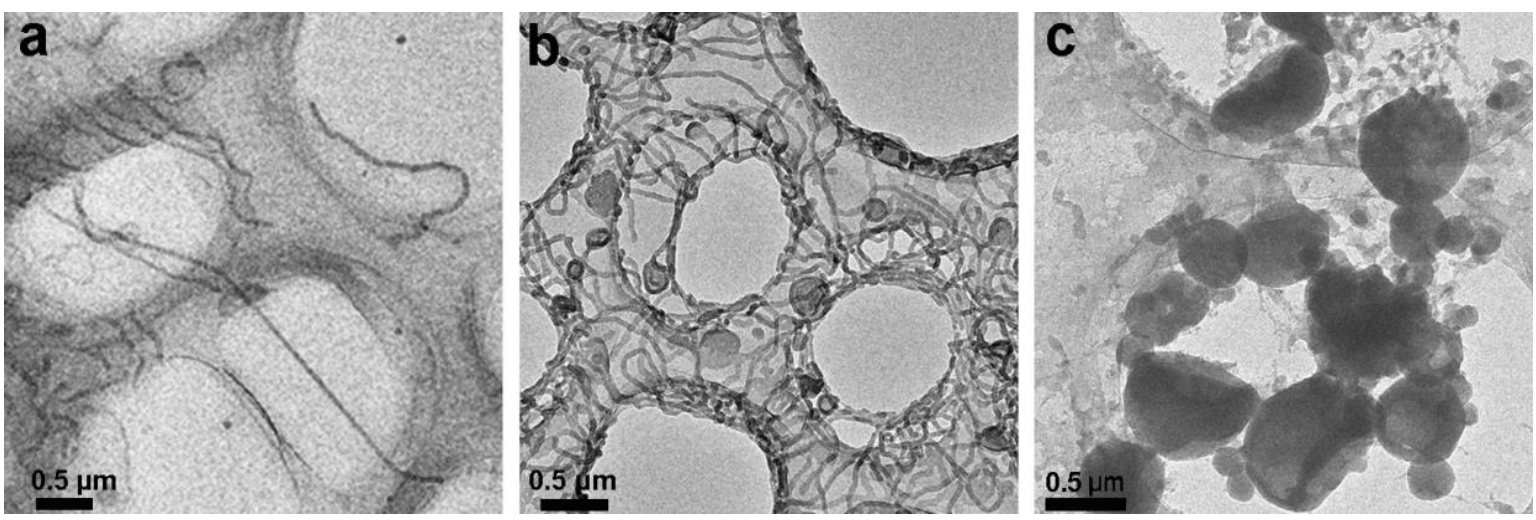

Figure S2 Cryogenic electron microscopy images of a) as-prepared PWs, b) after stored for 8 days, c) after 28 days. The medium is the MeOH/THF (1.5/1, v/v) mixed solvent. The results of the cryogenic TEM are consistent with those observed by TEM in a dry state, excluding the possibility that these morphologies were formed during the solvent evaporation. Thus, the TEM results used in this manuscript is reliable. 


\section{S6 Structure and Composition of the c-P4VP Nanogel}

\section{S6.1 More than $72 \%$ of the Pyridine Groups in c-P4VP Remained Unreacted}

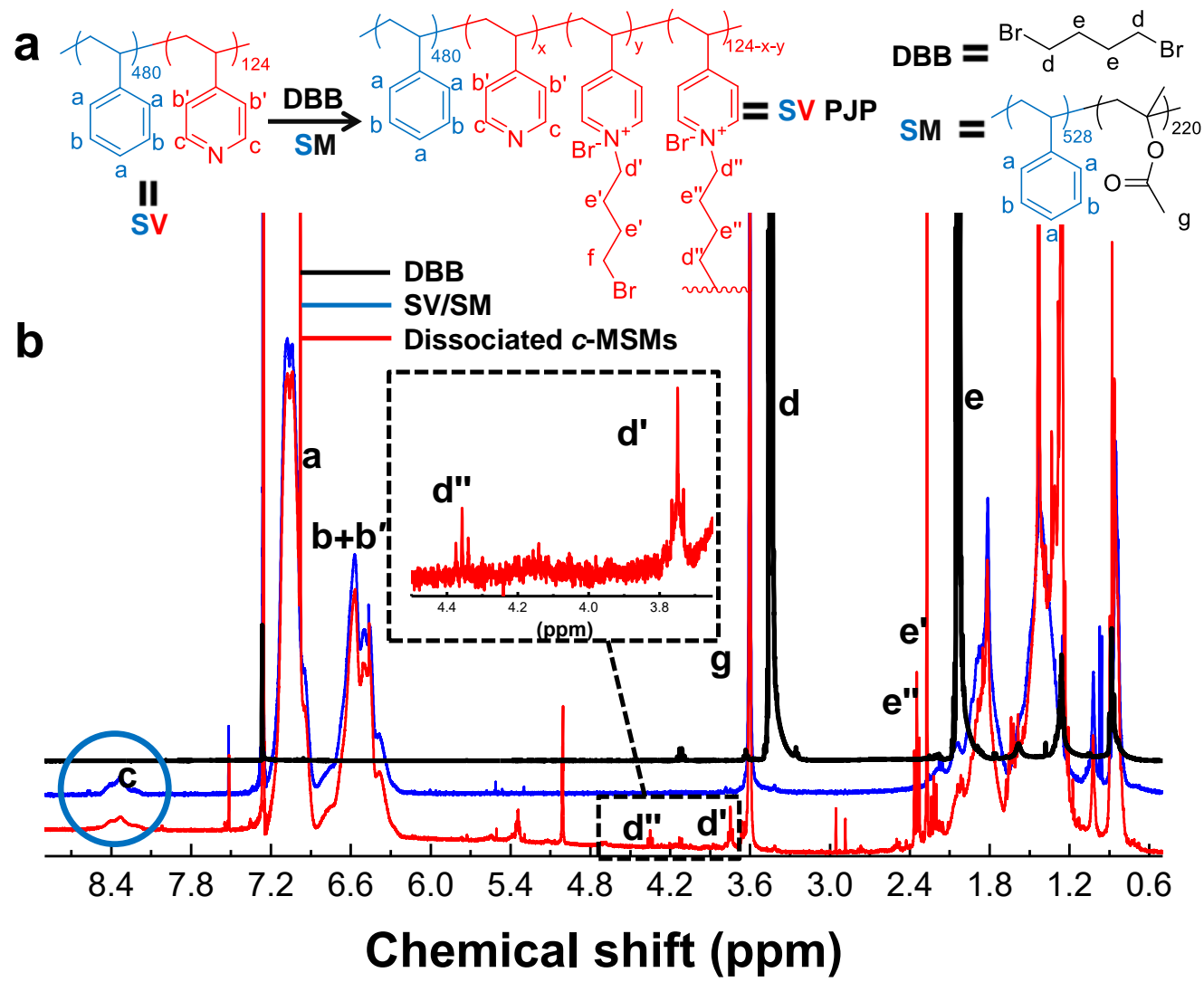

Figure S3. a) Cross-linking reaction between SV in the SV/SM MSMs and DBB. Only the P4VP chains were able to be cross-linked. b) ${ }^{1} \mathrm{H}$ NMR (CDCl ${ }_{3}$ spectra of DBB, SV/SM, and dissociated c-MSMs. The magnified peaks of $\mathbf{d}^{\prime}$ and $\mathbf{d}$ " were inserted. Each sample was dissolved in $\mathrm{CDCl}_{3}$, and the peak a was used as the internal standard.

When DBB reacts with the P4VP chains, there are two possible reactions, i.e., grafting DBB to one pyridine group and crosslinking DBB with two pyridine groups. Therefore, three kinds of pyridine groups are produced; the unreacted pyridine groups, the pyridine groups grafted by DBB, and pyridine groups cross-linked by DBB.

All the pyridine groups of SV/SM are the unreacted pyridine groups and have good mobility in $\mathrm{CDCl}_{3}$. Therefore, all the unreacted pyridine groups at peak $\mathbf{c}$ are detectable. As shown in Figure S3, compared with the integration area of peak c in SV/SM, the integration area of peak $\mathbf{c}$ in the dissociated c-MSMs is about $72 \%$ of that in SV/SM. That means that about $72 \%$ of the unreacted pyridine groups remain detectable relative to all the unreacted pyridine groups in SV/SM before cross-linking. After cross-linking, the $c$-P4VPs in the dissociated $c$-MSMs have both the unreacted pyridine groups and the reacted pyridine groups. The reacted pyridine groups are the quaternized pyridine groups that do not contribute to the signal at peak c. Moreover, the mobility of the unreacted pyridine groups near the cross-linking point is severely restricted. The unreacted pyridine signal of those unreacted pyridine groups near the cross-linking point cannot be detected. Only the signal of those unreacted pyridine groups that are far away from the crosslinking point can be detected since they have the good mobility. Therefore, there existed more than $72 \%$ of the unreacted pyridine groups relative to all the pyridine groups before cross-linking.

\section{S6.2 A Slightly Cross-linked and a Swollen Structure of $c$-P4VP}

Based on our previous study, ${ }^{[6]}$ when the P4VP chains were sufficiently cross-linked, the signal of the unreacted pyridine groups in the ${ }^{1} \mathrm{H}$ NMR spectrum disappeared completely due to the seriously restriction of the mobility of the pyridine groups located in the network. In the present work, $72 \%$ of the unreacted pyridine groups remain detectable, where the mobility of most of the unreacted pyridine groups is not restricted. Therefore, it indicates that $c$-P4VP is slightly cross-linked and has a loose structure.

\section{S6.3 A Considerable Number of 4VPs Grafted by DBB}

As shown in Figure S3, the spectrum of the dissociated $c$-MSMs contains both peak $\mathbf{d}^{\prime}$ and peak $\mathbf{d}^{\prime \prime}$. When DBB was grafted to the pyridine group, the relatively strong $\mathrm{Ar}-\mathrm{N}^{+} \mathrm{CH}_{2} \mathrm{CH}_{2} \mathrm{CH}_{2} \mathrm{CH}_{2} \mathrm{Br}$ peak (d') was found at 3.7-3.79 ppm. When DBB involved in the cross-linking of P4VP, the relatively weak Ar- $\mathrm{N}^{+} \mathrm{CH}_{2} \mathrm{CH}_{2} \mathrm{CH}_{2} \mathrm{CH}_{2} \mathrm{~N}^{+}$-Ar peak (d") was found at 4.33-4.77 ppm. Therefore, there existed a considerable number of DBB grafts, which are DBB molecules with one end reacted and connected with the pyridine groups while the other end unreacted. 


\section{S6.4 At Least $44 \%$ of the Pyridine Groups of c-P4VP Remained Unreacted after Storing for 28 days}

As mentioned in S6.1 that $c$-P4VP has more than $72 \%$ unreacted pyridine groups (relative to the pyridine groups before the cross-linking). In the rest reacted pyridine groups, some of them are grafted by DBB and some of them are involved in the crosslinking of P4VP by one DBB reacting with two pyridine groups in P4VP. Therefore, there have less than $28 \%$ pyridine groups (relative to the pyridine groups before the cross-linking) grafted by DBB. One end of DBB is reacted with the pyridine group while the other end of DBB is unreacted.

With increasing of the storing time, the unreacted end of the DBBs in the $c$-P4VP would react with the unreacted pyridine groups to further cross-link the P4VP. Supposing that the unreacted end of the DBBs all react with the unreacted pyridine groups in $c$-P4VP there still exist more than $44 \%$ unreacted pyridine groups (relative to the pyridine groups before the cross-linking).

\section{S7 Self-driven Morphological Transition from Spherical Micelles to Vesicles}

The self-driven morphological transition from spherical micelles to vesicles was also realized. The spherical micelles were obtained by co-assembling of PJPs and SM ( W $\left._{S V} / \mathrm{W}_{S M}=1.1: 1\right)$ as described in the experimental section. The obtained spherical micelles were monodisperse with an average diameter of $44 \mathrm{~nm}$ (Figure S4), with PS as the core and $c$-P4VP/PMMA as the mixed shell. After storing the sealed suspension for 40 days, the spherical micelles were converted to vesicles.

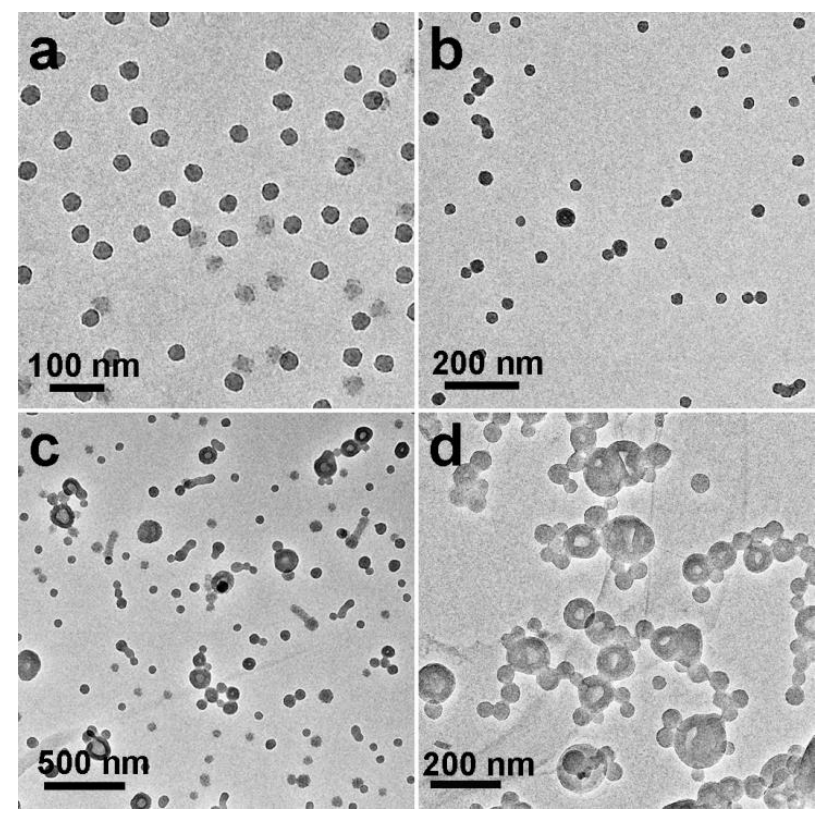

Figure S4. TEM images of spherical micelles co-assembled by PJPs and SM ( $\left.w_{S V} / w_{S M}=1.1: 1\right)$ and their morphological transition from spherical micelles to vesicles. a) As-prepared spherical micelle, b-d) morphological transition from spherical micelles to spherical micelle/short PW/vesicle mixture and then to all vesicles after sealed for b) 10 days, c) 20 days, and d) 40 days. The medium is the MeOH/THF $(1.5 / 1$, v/v) mixed solvent. 


\section{S8 Frozen PS Core in the PWs}
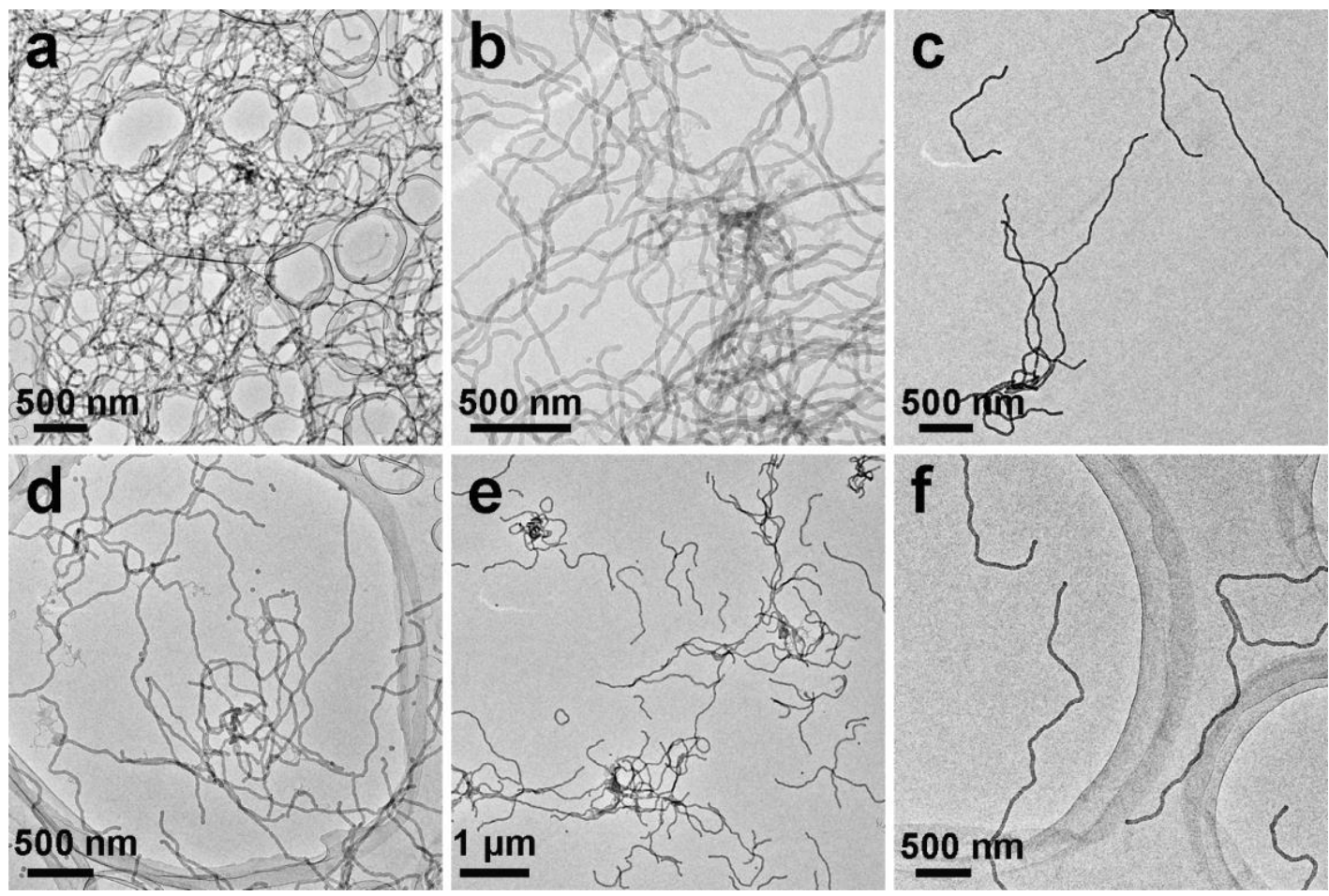

Figure S5. PWs sealed for a) 3 days, b) 8 days, c) 15 days, d) 22 days, e) 28 days, and f) 139 days. The medium is the MeOH/THF (24/1, v/v) mixed solvent. The worm-to-vesicle morphological transition that would happen when the MeOH/THF volume ratio is $1.5 / 1$ was completely prohibited when the volume ratio is $24 / 1$. This fact reveals that the PS core in the mixture solvent at the MeOH/THF volume ratio of $24 / 1$ is frozen.

\section{S9 Existence of Excess TFAs has No Influence on the Results of the Present Study}

TFA molecule 1) is highly soluble, 2) has a low boiling point, and 3) is immisible with PS. The reason why the existence of excess TFAs has no influence on the result of the present study is decribed below.

1) TFA molecules are highly soluble in the MeOH/THF $(24 / 1, \mathrm{v} / \mathrm{v})$. It is molecularly dissolved in the medium, which cannot be detected by DLS measurement. Hence, the existence of excess TFAs in the suspension does not influence the result of DLS measurement.

2) TFA has a low boiling point $\left(72^{\circ} \mathrm{C}\right)$ and therefore easily evaporate in vacuum. The residual TFAs in the TEM sample can easily volatilize of in the high vacumm environment during TEM sample preparation and observation. Therefore, the existence of excess TFAs in the suspension does not influence the result of TEM observation.

3) As reported, ${ }^{[6,7]}$ in the MeOH/THF $(24 / 1, v / v)$, TFAs can be complexed with $c$-P4VP via the strong hydrogen bonding interaction between the carboxyl groups of TFAs and the unreacted pyridine groups in $c$-P4VP. Moreover, the control experiment was conducted as below. In the vesicle suspension of PS- $b$-PMMA in mixed solvent of MeOH/THF (24/1, v/v), the same concentration of highly excess TFAs (6\%) was added and the mixture was stirred for $24 \mathrm{~h}$. It was found that the vesicles did not have any morphological change. This indicates that the highly excess TFAs do not interact with PS or PMMA to change the morphology of vesicles. Additionally, the same concentration of highly excess TFAs $(6 \%)$ was added in the core-frozen micelles suspension coassembled by PS- $b$-PMMA/PS- $b$-P4VP (MeOH:THF $=24 / 1, \mathrm{v} / \mathrm{v})$. After the mixture was stirred for $24 \mathrm{~h}$, the micelles did not have any morphological change either. Athough the highly excess TFAs interact with the P4VP chains on the shell, they still can not change the micelles morphology.

As is known from the above discussion, there is no interaction between PS and TFA; the PS and TFA are highly immiscible. The excess TFA molecules have no influence on the behavior and structure of assembles and also the TEM and DLS results. In the present work, highly excess TFAs were added to the system (MeOH:THF = 24/1, v/v). The molar ratio of TFA/pyridine was 1000:1. TFAs complexed with $c$-P4VP via hydrogen bonding interaction between the carboxyl groups of TFAs and the unreacted pyridine groups in c-P4VP. As discussed in S6.1, c-P4VPs in the PWs have more than $72 \%$ unreacted pyridine groups. The $c$-P4VP nanogels in the shells of assemblies were completely complexed by excess TFA. This effectively increased the size of the $c$-P4VP nanogels and the repulsions between the shell forming blocks. Thus the core-frozen PWs and vesicles broke into fragments. The remaining uncomplexed TFA molecules has no influence on the behavior and structure of assembles. 


\section{References}

[1] A. Blanazs, S. P. Armes, A. J. Ryan, Macromol. Rapid. Comm. 2009, 30, 267-277

[2] S. Y. Liu, S. P. Armes, J. Am. Chem. Soc. 2001, 123, 9910-9911.

[3] Z. Zhang, H. D. Li, X. Y. Huang, D. Y. Chen, ACS Macro Lett. 2017, 6, 580-585

[4] Z. Zhang, C. M. Zhou, H. Y. Dong, D. Y. Chen, Angew. Chem. Int. Ed. 2016, 55, 6182-6186.

[5] Y. Habata, A. Taniguchi, M. Ikeda, T. Hiraoka, N. Matsuyama, S. Otsuka, S. Kuwahara, Inorg. Chem. 2013, 52, 2542-2549.

[6] a) L. Cheng, G. L. Hou, J. J. Miao, D. Y. Chen, M. Jiang, L. Zhu, Macromolecules 2008, 41, 8159-8166; b) H. S. Peng, D. Y. Chen, M. Jiang, Langmuir 2003, 19, 10989-10992.

[7] H. S. Peng, D. Y. Chen, M. Jiang, J. Phys. Chem. B 2003, 107, 12461-12464 